\title{
Invisible Hands Behind 3.5 GHz Spectrum Sharing
}

\author{
Liu CUI* and Martin WEISS ${ }^{\dagger}$ \\ *Department of Computer Science, West Chester University \\ **School of Information Sciences, University of Pittsburgh
}

\begin{abstract}
There has been considerable discussion surrounding the barriers to spectrum sharing in the literature. Among those is the 'trust gap' that exists, according to the PCAST report. Trust is a complex human construct that significantly includes risk. In this paper, we examine the risks faced by the different user classes proposed by the FCC for sharing in the $3.5 \mathrm{GHz}$ band. We argue that the "invisible hands" of spectrum sharing in this band is the balance between spectrum sharing gain and associated risks. We find that both gains and risks can be linked to the distance between incumbents' systems and Citizen Broadband Radio Services (CBRS)' systems. The risk portfolio is linked to spectrum rights that each tier has, since the rights they have determine risks and risk mitigation strategies. We further propose a model to calculate spectrum sharing utilities for different tiers. The optimized utility determines the distance between incumbents and CBRS systems.
\end{abstract}

Key words: spectrum sharing, $3.5 \mathrm{GHz}$

\section{Introduction}

On April 21, 2015, Federal Communications Commission (FCC) released the Report and Order (R\&O) for the $3.5 \mathrm{GHz}$ band [FCC (2015)]. In this document, FCC describes the creation of "Citizens Broadband Radio Service" (CBRS) for this band, which will be implemented by allowing non-federal users to share spectrum with incumbents. Incumbents include Department of Defense (DoD) Radar Systems in 3550-3650 MHz band, Fixed Satellite Services (FSS) and grandfathered terrestrial wireless operations in $3650-3700 \mathrm{MHz}$. The sharing arrangement between federal and non-federal usage will take place under a three-tiered sharing framework enabled by a Spectrum Access System (SAS). The highest tier, incumbent users, receives interference protection from other users. The CBRS itself contains two tiers: Priority Access Licenses (PALs) and General Authorized Access (GAA). PAL holders receive interference protection from GAA applications. GAA users receives no interference protection from other CBRS users.

\footnotetext{
* lcui@wcupa.edu

$\dagger$ mbw@pitt.edu
} 
This three tier spectrum sharing arrangement only provides a spectrum sharing framework. It does not promise a future with widely adopted spectrum sharing. Thus, promoting spectrum sharing in $3.5 \mathrm{GHz}$ is an important question after FCC's rulemaking. Otherwise, $3.5 \mathrm{GHz}$ may face the same situation as TVWS, which opened unlicensed access in 2008 but has not been widely utilized Robyn et.al. (2015).

An underlying reason for the slow adoption of spectrum sharing is that there are risks associated with this approach. Incumbents are understandably concerned about potential interference, so they defend their rights to licensed frequencies. Potential CBRS users are uncertain about the regulation and spectrum environment (i.e., their usage rights as well as the collective action rights |Weiss et.al.(2015)]) so both service providers and device manufacturers may be cautious about spectrum sharing in $3.5 \mathrm{GH}_{2}^{3}$.

In order to promote spectrum sharing in $3.5 \mathrm{GHz}$, it is essential that risk management strategies for each usage tier in $3.5 \mathrm{GHz}$ be developed. Since the rights, missions, applications, etc. vary with usage tier. Spectrum users of each tier face different risks than users of other tiers and thus need specific strategies to cope with these risks. To address this, we analyze spectrum sharing in $3.5 \mathrm{GHz}$ with the specific purpose of clarifying the rights and quantifying risks for each tier as well as identifying appropriate risk management. Finally, we analyze the trade off between benefits associate spectrum sharing and costs associate with risk. This trade off is the invisible hand behind spectrum sharing that determines the size of exclusion and protection zone, and applications in CBRS.

\section{Rights in $3.5 \mathrm{GHz}$}

Risks and rights may be closely coupled with one another when rights are poorly defined. So we begin by introducing the users of $3.5 \mathrm{GHz}$ and their de jure and de facto rights. We begin by a brief reprise of the rights framework discussed in [?, Weiss et.al.(2015)]

In her work, the Nobel prize winning economist Elinor Ostrom attended to the rights and governance of so-called common pool resource systems. These are systems in which one user's consumption diminishes another user's consumption opportunities (i.e., the uses are "subtractible" or "rivalrous") and in which exclusion is difficult or costly. [Weiss et.al.(2015)] argued that spectrum best fits this type of good under the current technology endowment. They also modified Ostrom's rights framework as shown in Table 1. This table describes the rights endowment of five different user types for five different types of rights. The first two rights (i.e., Reception and Transmission) can be considered usage rights because they relate to the operation of the how the system is used, while the

\footnotetext{
${ }^{3}$ In his keynote address to IEEE DySPAN in 2015, Dr. Ranveer Chandra of Microsoft Research indicated that ASICs for TVWS had been designed, but that their manufacture was deferred until sufficient demand for TVWS devices could be demonstrated.
} 
remaining three are referred to as collective action rights and refer to the design of the rights system. In particular, management rights refer how the resource is used and managed, exclusion rights refer the determination of who has access to the resource, and alienation rights refer to the rights to transfer any of the other rights.

In spectrum sharing systems, interference is inevitable, which introduces risk. Thus, we briefly discuss interference from the perspective of this framework. In a prior paper, we examined the possibility of creating a right out of what might otherwise be considered an 'externality ${ }^{4}$ ' |Weiss and Cui(2012)]. Referring to the framework in Table 1 interference occurs when a signal from one authorized sender impinges on another authorized receiver ${ }^{5}$ In general, this occurs as an unexpected result of the management regime that was determined through the exercise of collective action rights, even if it is the result abnormal propagation circumstances.

\begin{tabular}{l|l|l|l|l|l|}
\cline { 2 - 6 } & Full & Prop- & Auth. & Auth. & Auth. \\
& owner & rietor & claimant & sender & rcvr \\
\hline \hline Reception & $\mathrm{X}$ & $\mathrm{X}$ & $\mathrm{X}$ & $\mathrm{X}$ & $\mathrm{X}$ \\
Transmission & $\mathrm{X}$ & $\mathrm{X}$ & $\mathrm{X}$ & $\mathrm{X}$ & \\
Management & $\mathrm{X}$ & $\mathrm{X}$ & $\mathrm{X}$ & & \\
Exclusion & $\mathrm{X}$ & $\mathrm{X}$ & & & \\
Alienation & $\mathrm{X}$ & & & & \\
\hline
\end{tabular}

Table 1. Distribution of rights by user type

With this background in mind, we examine the stakeholders in the $3.5 \mathrm{GHz}$ band.

\subsection{Incumbents}

The current spectrum allocation in $3.5 \mathrm{GHz}$ band is already very complicated. Through the collective action processes of the CSMAC and FCC, the entire 3550-3700 MHz was divided into two sub-bands: $3550-3650 \mathrm{MHz}$ and $3650-3700$ MHz. A two tiered hierarchical usage rights scheme was developed for each subband (an exercise of the management rights) that are called primary usage and secondary usage. Further, both federal and non-federal usage was permitted under both primary and secondary usage. We begin with primary usage in the 3550-3650 MHz sub-band: The R\&O granted primary usage rights to limited non-federal Fixed Satellite Service (FSS) if they existed prior to the effective date of the R\&O. As well, federal fixed and mobile (except aeronautical mobile)

\footnotetext{
${ }^{4}$ In economics, an externality is a cost or benefit that affects a party who did not choose to incur that cost or benefit (Source: Wikipedia).

${ }^{5}$ Rogue transmissions are from unauthorized senders and so fall outside of this framework.
} 
radar systems were given primary usage rights. The $\mathrm{R} \& \mathrm{O}$ granted subordinate (i.e., secondary) usage rights to Federal Radio Location Services (RLS) and certain low power non-Federal applications.

$3650-3700 \mathrm{MHz}$ is less crowded than $3550-3650$ sub-band. Here, primary usage rights are granted to some Federal RLS sites and ships for radar. Secondary usage rights are granted to wireless broadband services.

Superior (i.e. primary) usage rights imply the right to receive without interference from authorized suboardinate (secondary) users. Stated differently, this means that incumbents have the highest priority in spectrum access. But this classification does not address interference between different rights holders of the same class. That is, do some users have super-primary rights? Some DoD radar systems including ground-based, shipborne, and airborne platforms, which are used in conjunction with weapons control systems, may cause interference even to other primary users. In addition, incumbents with primary usage rights have the right to deploy both fixed and mobile transmitters that in line with their mission.

\subsection{Citizens Broadband Radio Service}

The authorization of CBRS rights is limited by inferiority to the primary users' rights. Further, the $\mathrm{R} \& \mathrm{O}$ envisions two further tiers: Priority Access Licensees (PALs) and General Authorized Access (GAA). In the framework of Table 1. PAL usage rights are superior to GAA usage rights.

Both classes of users have collective action rights through the FCC process. Of these, management rights are the most dynamic since the licensing procedures for PALs addresses exclusion rights. One important challenge ex ante in the domain of exercising management rights is the question of appropriately balancing the spectrum allocation between PALs and GAA. This must be accomplished ex ante since PALs will be auctioned (see below). PALs need to be sufficiently attractive to attract bids while simultaneously fostering a robust GAA ecosystem for innovation.

Considering the comments and suggestions from different stakeholders, the FCC concluded that a maximum of $70 \mathrm{MHz}, 7$ channels (10 MHz each), should be reserved for PALs in any given license area at any time. In addition, every PAL can aggregate up to four channels in any given license area to encourage competition. The remainder of the available frequencies is made available for GAA usage. The definitions are as follows:

PALs Any prospective licensee who meets basic FCC qualifications is eligible for PALs. All applicants for PALs must demonstrate their qualification to hold an authorization and demonstrate how a grant of authorization would serve the public interest. Census tracts is the license size for PALs. PALs have three-year non-renewable license terms - with the ability to aggregate up to six years up-front. Finally, PALs will be assigned by competitive bidding.

GAA FCC reasoned that a license-by-rule licensing framework would allow for rapid deployment of small cells by a wide range of users, including consumers, 
enterprises, and service providers, at low cost and with minimal barriers to entry.

GAA users may only use FCC certified Citizens Broadband Radio Services Devices (CBSDs) and must register with the SAS. Consistent with rules governing CBSDs, devices operating on a GAA basis must provide the SAS with all information requirement by the rules, including operator identification, device identification, and geo-location information, upon initial registration and as required by the SAS. Moreover, only fixed CBSDs are allowed at this stage.

We now translate this policy into the rights framework of Table 1. According to the $\mathrm{R} \& \mathrm{O}$,

"To ensure that essential federal radiolocation systems operating in the band continue their operations without impact from the sharing arrangements, we are prohibiting CBSDs from causing harmful interference to, or claiming protection from, federal stations aboard vessels (shipborne radars) and at designated groundbased radar sites. In addition, authorized users of CBSDs must not claim protection from airborne radars and airborne radar receivers must not claim protection from CBSDs operating in the Citizens Broadband Radio Service."

The notion of "interference protection" in this exerpt from the R\&O means that the transmission rights of CBSDs are subordinate to the receiving rights of the incumbent users. Furthermore, this management regime does not limit Federal RLS transmission rights in order to preserve CBSDs reception rights. That is, they do not have the right of interference protection from incumbents. Transmission and reception rights are similarly organized within CBRS so that PALs' reception rights are superior to GAA's transmission rights (i.e., they have the right of interference protection from GAA users); like RLS, GAA users have no rights of interference protection from incumbent and other Citizens Broadband Radio Service users. This management and exclusion regime is encoded in a Spectrum Access System (SAS), which is a spatial database that is used to implement the rights regime described the the previous paragraph in real time.

\section{Invisible Hands behind Spectrum Sharing in $3.5 \mathrm{GHz}$}

In 1776, Adam Smith mentioned "the invisible hand" in his classic book "The Wealth of Nations" to describe the natural force that guides free market capitalism through competition for scare resources. He pointed out that no regulation of any type would be necessary to ensure the mutually beneficial exchange of good and services in a free market, since the "invisible hand" (the intent that each participant tried to maximize self-interest) will lead to the most mutually beneficial manner.

There is an "invisible hand" in spectrum sharing in $3.5 \mathrm{GHz}$ as well. Although the exclusion and protection zone is currently determined by incumbents and 
regulators, a boundary can be determined voluntarily when considering both the benefit of spectrum sharing and cost associated with risks. For example, two extreme cases may exist: (1) when advanced technology can control all transmitters and monitor spectrum usage in real-time at low cost, the exclusion zone will be very small if it even exists; (2) when it is difficult to control transmitters and monitor spectrum usage in a timely manner and cost effective way, the exclusion zone will be very large.

\subsection{Risks in $3.5 \mathrm{GHz}$}

According to TAC, there are three categories to evaluate risks: (1) corporate metrics, (2) service metrics, (3) RF metrics. Coporate metrics include the ability to complete a mission, loss in revenue and profits. Service metrics measures the availability and quality of the service. RF metrics measure signal to interference and noise ratios, absolute interfering signal level, etc [FCC (2015)].

In this paper, RF metric is evaluated by interference estimation described in section 3.1, service metrics is evaluated by spectrum access opportunities described in section 3.1 and the corporate metric is evaluated by profit that gained wireless service providers described in section 3.2. Moreover, as a wireless service provider, they do not passively accept risks. Instead, users with different rights have various strategies to cope with risks. Section 3.1 describes risk mitigation strategies for each tier.

Interference Estimation The primary RF risk for incumbents is that CBSDs may bring harmful interference to their systems, which negatively impacts their ability to carryer out their mission. Similarly, CBSDs also have risks in receiving interference. Specifically, PALs may receive interference from incumbents, and GAAs may receive interference from both incumbents and PALs. We determine the potential interference that comes from a higher tier to the lower tier. For example, PALs estimate the interference that comes from incumbents, and GAAs estimate the interference that comes from both incumbents and PALs.

Although we don't have the information on transmission power level, we can follow the reverse engineer the NTIA exclusion zone calculations. First, we determine the maximum interference level that PALs and GAAs can accept. We assume that PALs can accept interference level $\left(I^{P}\right)$ up to $-20 \mathrm{dBm}$ and GAAs can only accept interference level $\left(I^{G}\right)$ up to $-30 \mathrm{dBm}$. Then, we adopt the same path loss model $L_{p}$ that NTIA used for calculating exclusion zones. Finally, the acceptable incumbent transmission power $\left(X^{P}\right)$ is determined as $X^{P}=I^{P}+L_{p}$ and $X^{G}=I^{G}+L_{p}$ for PALs and GAAs respectively.

$$
\begin{aligned}
& L_{p}= 69.55+26.16 \log f-13.82 \log h_{b}-a\left(h_{m}\right) \\
&+\left(44.0-6.55 \log h_{b}\right) \log d \\
& a\left(h_{m}\right)=\left\{\begin{array}{lc}
3.2\left(\log \left(11.75 h_{m}\right)\right)^{2}-4.97, & \text { L City } \\
\left(1.1 \log (f-0.7) h_{m}-1.56 \log f+0.8, \text { M } /\right. \text { S City }
\end{array}\right.
\end{aligned}
$$


Therefore, the probability of interference is determined as:

$$
P\left(P_{t}>X^{i}\right)=1-\frac{1}{2}\left[1+\operatorname{erf}\left(\frac{x-\mu}{\sigma \sqrt{2 \pi}}\right)\right], \quad i=P, G
$$

when we assume the transmission power level $\left(P_{t}\right)$ follows a normal distribution, $P_{t}=\frac{1}{\sigma \sqrt{2 \pi}} e^{-\frac{(x-\mu)^{2}}{2 \sigma^{2}}}$, with mean $\mu$ and deviation $\sigma$.

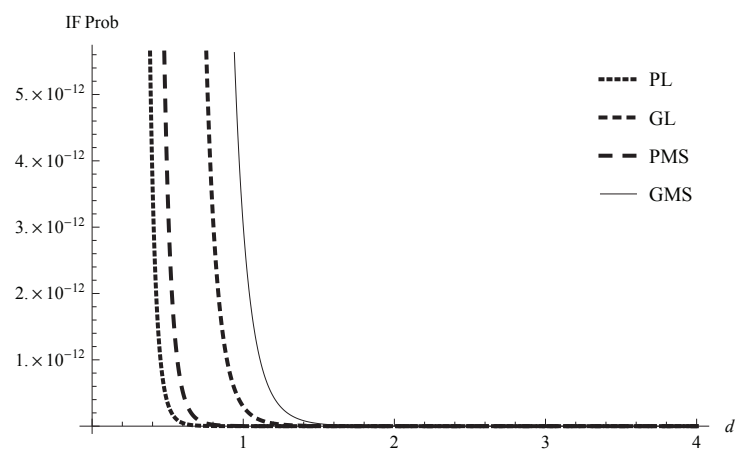

Fig. 1. Probability of receiving interference from incumbents $\sigma=10$

Fig, 1 and Fig, 2 show the probability of interference for situations with respect to the distance between transmitters and receivers, CBSDs' interference threshold, and path loss model in large and medium/small city. We assume that $\mu=82 \mathrm{dBm}, \sigma=10$ and $\sigma=100$ in Fig 1 and Fig 2 respectively. It is clear that when the distance $(d)$ between transmitters and receiver stays the same, PALs in a large city (PL) have the lowest probability of interference because PALs have less sensitivity to interference than GAAs and the path loss in a large city is more severe than a medium/small city. When the path loss decreases (in the medium/small city case), the probability of interference increases in PMS. A similar pattern can be recognized for GAAs. Further, GAAs have higher probability of interference in the same geographic region than PALs, since GAA's interference threshold is higher than PALs. Moreover, the probability of interference decreases with increases of $d$, due to the path loss factor. Compare Fig 1 and Fig. 2. when $\sigma$ increase from 10 to 100, the transmission power is less centralized to the mean. Therefore, the probability of interference increases.

Spectrum Access Opportunities Estimation According to the R\&O, all frequency bands that are not occupied by incumbents and PALs can be utilized by GAAs. In the ideal case, GAAs have at least $80 \mathrm{MHz}$ in any geographic areas outside the exclusion zones (recall that the total shareable frequency is $150 \mathrm{MHz}, 70 \mathrm{MHz}$ is allocated to PALs, so the remaining $80 \mathrm{MHz}$ can be used for GAAs.) Let us calculate the spectrum access opportunities for GAAs on 


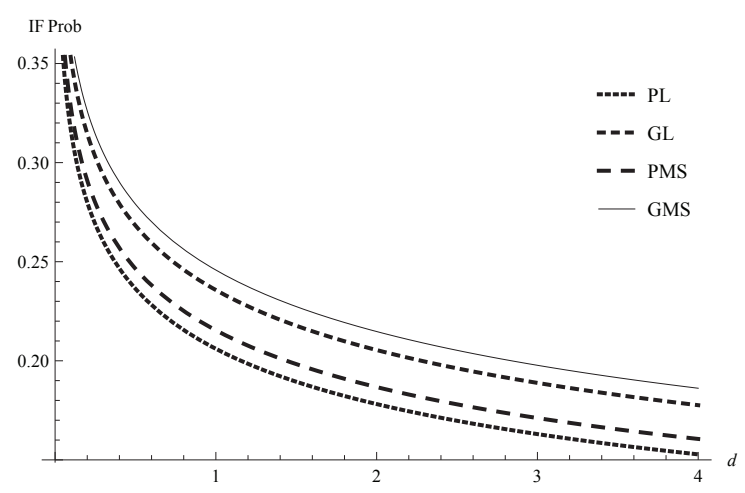

Fig. 2. Probability of receiving interference from incumbents $\sigma=100$

those $80 \mathrm{MHz}$. It is assumed that GAAs can perfectly detect each other and there is no interference from PALs and incumbents.

Since GAAs are controlled by a SAS on a FCFS base, we adopt a queueing model to quantify the probability of the spectrum access opportunity. It is assumed that GAAs arrival process follows a Poisson distribution with mean $\lambda$, and the departure process follows exponential distribution with mean $\mu$. It is further assumed that the capacity of the system is 8 channels with $10 \mathrm{MHz}$ bandwidth. Consequently, we adopt $M / M / C$ queue with $C=8$.

The most important metric is the probability of waiting $\left(P_{Q}\right)$. Thus, the probability of spectrum is available is $\left(1-P_{Q}\right)$.

$$
P_{Q}=\sum_{i=C}^{\infty} P_{i}=\frac{(C a)^{C}}{C !} \frac{1}{1-a} P_{0}
$$

where, $C$ is the total number of available channel, $a=\frac{\lambda}{\mu}$ and

$$
P_{0}=\left(\sum_{i=0}^{C-1} \frac{(C a)^{i}}{i !}+\frac{(C a)^{C}}{C !(1-a)}\right)^{-1}
$$

In the future, we will tailor this probability according to different applications' requirement, such as elastic vs inelastic services by using metrics like mean waiting time $(\bar{W})$ and probability that waiting time is greater than threshold $t$ $(P(W>t)$.

Coping with Risks Under a subordinate rights structure, spectrum users with different priority have different risk metrics and risk measurement/protection methods. Spectrum users with higher priority have the right to protect their own services. However, spectrum users with lower priority can only estimate risks and make informed decision accordingly. 


\section{Protection}

There are many ways to protect the system from interference: geographic separation, frequency separation, time separation are three dominate approaches. Since CBRS shares spectrum with current users, incumbents focus on geographic and time separation. Here, risk protection starts with the incumbents' own requirements, such as Signal to Noise and Interference Ratio (SNIR). Then, incumbents determine exclusion (and protection) zone where no one else can operate based on PALs and GAAs allowable transmission power limits as well as an appropriate path loss model. Even outside the exclusion zones, CBSDs can only transmit when incumbents are absent.

PALs have interference protection rights over GAAs and focus on frequency and time separation. This means that PALs have dedicated frequency bands where no other CBSDs can operate. The time seperation is implemented by the SAS through explicit grants of GAA's transmission rights, which, in turn, is based on the PAL's traffic.

\section{Estimation}

For CBRS, although they do not have the right of interference protection from incumbents, they can actively cope with risks by estimating potential risks before hand and then making informed decisions and using flexible management. CBRSs may have different goals; for example, hospitals and public safety provide life critical services that can hardly be measured by money while commercial services can be evaluated by revenue and customer satisfaction.

Accordingly, we will estimate risks for Citizens Broadband Radio Services from the QoS perspective and then link it to potential revenue. Two risk metrics are applied in analyzing QoS.

- Probability of interference. In this metric, spectrum users calculate the probability that they may be interfered by users with higher priority.

- Probability of getting spectrum without waiting. This risk metric only applies to GAAs, since they do not have spectrum reservation.

When we link QoS to potential revenue, we assume that the maximum revenue that CBSDs can earn occurs when there is no interference and spectrum is available all the time. We assume that the potential revenue that CBSDs can earn linearly decreases with probability of interference and the probability of waiting for available spectrum.

\subsection{Benefit for CBRS}

The invisible hands behind the spectrum sharing is to maximize the overall benefit getting from spectrum sharing in $3.5 \mathrm{GHz}$. We assume that the benefit for spectrum sharing with PALs $\left(U^{P}\right)$ is calculated as the maximum benefit per user $\left(u^{p}\right)$ times number of users/devices covered by PALs $\left(N_{c}^{p}\right)$. Moreover, this maximum value can be achieve when there is no interference from incumbents $\left(1-P^{I}(i)\right)$ as shown in equation 6

$$
U^{P}=u^{p} \times N_{c}^{p} \times\left(1-P^{I}(i)\right)
$$


GAA are more poorly situated than PALs, since they accept interference from both incumbents and PALs. Also, they cannot reserve spectrum, since the SAS allocates frequency bands to GAA on a FCFS basis. In other words, GAAs can provide services when spectrum is available. As a result, there are three risk metrics for GAAs: the probability of getting interference from incumbents $P^{I}(i)$, the probability of getting interference from PALs $P^{G}(i)$, and the probability that a frequency band is not available right away $P_{Q}$. The benefit of spectrum sharing in GAA $\left(U^{G}\right)$ equals the maximum benefit per user/device $\left(u^{g}\right)$ times number of users/devices covered by GAA $\left(N_{c}^{g}\right)$, and this benefit can only be achieved when spectrum is available without interference $\left(1-P^{I}(i)\right)\left(1-P^{P}(i)\right)\left(1-P_{Q}\right)$ as shown in equation 7 .

$$
U^{G}=u^{g} \times N_{c}^{g} \times\left(1-P^{I}(i)\right) \times\left(1-P^{P}(i)\right) \times\left(1-P_{Q}\right)
$$

According to NTIA exclusion zone calculation, the population density decreases with the increase of exclusion zone radius $(d)$. Therefore the $N_{c}^{p}$ and $N_{c}^{g}$ are a function of exclusion zone radius.

$$
N_{c}^{i}=\frac{1}{d} \times \pi r_{i}^{2}, \quad i=p, g
$$

In order to calculate benefit of spectrum sharing, we need to start by estimating risks in spectrum sharing (illustrated in section 3.1 and section 3.1).

\section{Numerical Results}

In this section, we discuss some representative figures that demonstrate the invisible hands, i.e., the tradeoff between benefit and cost, that determine spectrum sharing. In Fig 3 the assumption is that user/device density decreases with the exclusion zone radius $(d)$. Although the interference also decrease with the increase of exclusion zone radius, density in this case is the dominate factor in determining the utility for PALs. Therefore, the smaller the $d$ is, the higher the utility.

Fig, 4 assumes the user/device density does not change according to the exclusion zone radius. Device to Device (D2D) communication networks can be one example of this scenario. Clearly, users have higher utility when they are away from incumbents, since the probability of interference from incumbents decreases. Further, utilities when $\sigma 1$ is applied is higher than utilities under $\sigma 2$, since the interference level in $\sigma 1$ is higher than $\sigma 2$. GAAs utility shows the same trend with lower utility value.

\section{Conclusion}

There is currently a great deal of interest in spectrum sharing in the $3.5 \mathrm{GHz}$ band. Success in this band is likely to encourage spectrum sharing in other bands, 


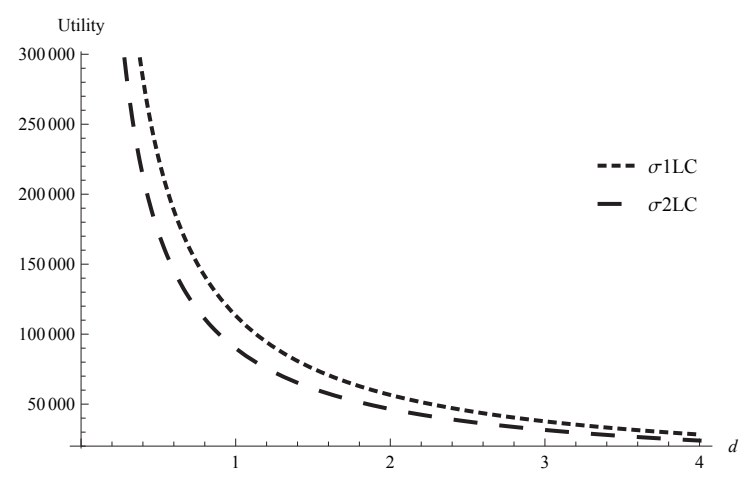

Fig. 3. PALs utility with changing user/device density

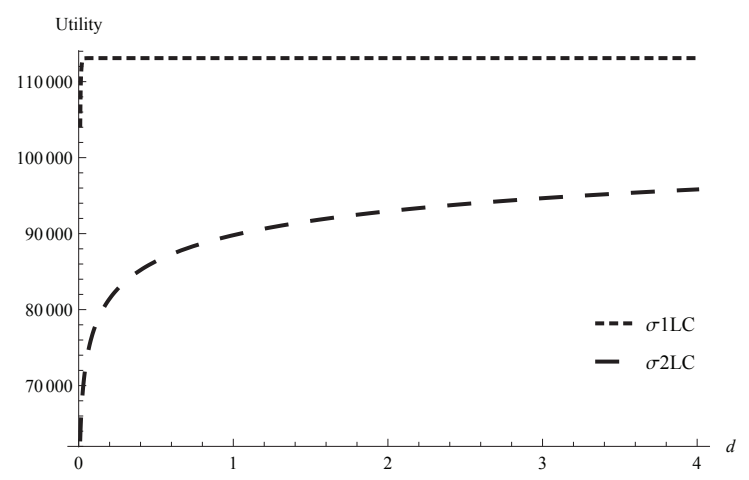

Fig. 4. PALs utility with constant user/device density

so an important challenge is learn how to incentivize spectrum sharing under this three tier spectrum sharing prototype while protecting spectrum users' systems and investment. This is complicated by the presence of risk because the costbenefit calculus becomes more complex. In this paper, we sought to identify and quantify the risks faced by the different classes of users users in $3.5 \mathrm{GHz}$ band as a first step to support users' decisionmaking. We did not address the impact of risk mitigation strategies, which change with the rights that different spectrum users hold.

For Adam Smith, the "invisble hand" guided markets in equilibrium. In the case of spectrum sharing, the "invisible hand" cautions users from investing because of the risks they face. These risks loom largest in the absence of experience, so quantifying and modelling the risks is a first step toward developing risk mitigation strategies that should cause the "invisible hand" to facilitate transactions rather than stop them. 


\section{Acknowledgement}

This work was supported by the US National Science Foundation under grant 1247546.

\section{References}

FCC (2015). Commission FC, et al (2015) Amendment to the commission's rules with regard to commercial operations in the band 3550-3650 mhz. FCC Gaussian noise Docket (12-354)

FCC (2015). FCC (2015) A case study of risk-informed interference assessment: Metsat/lte coexistence in 1695-1710 mhz

Robyn et.al. (2015). Robyn D, Jackson C, Bazelon C (2015) Unlicensed operations in the lower spectrum bands: Why is no one using the tv white space and what does that mean for the fcc?s order on the $600 \mathrm{mhz}$ guard bands? URL http://papers. ssrn.com/sol3/papers. cfm?abstract_id=2587528

Weiss and Cui(2012). Weiss MB, Cui L (2012) Spectrum trading with interference rights. In: Cognitive Radio Oriented Wireless Networks and Communications (CROWNCOM), 2012 7th International ICST Conference on, IEEE, pp 135-140

Weiss et.al.(2015). Weiss MB, Lehr WH, Acker A, Gomez MM (2015) Socio-technical considerations for spectrum access system (sas) design 